\title{
THE ROLE OF THE MICROBIOME IN RESPIRATORY DISEASE: ALLERGIC RHINITIS
}

\author{
Arundeep Singh ${ }^{1}$, Kirsten Kloepfer ${ }^{2}$ \\ Indiana University School of Medicine ${ }^{1}$; Indiana University School of Medicine, Division of \\ Pulmonary, Allergy, and Sleep Medicine ${ }^{2}$
}

\section{Background:}

Allergic rhinitis is one of the most common disorders affecting patients in the United States and developed and developing countries. Currently, no effective methods of prevention or effective cures exist for allergic rhinitis. This is due to unanswered questions regarding the etiology of allergic sensitization and the development of symptoms after sensitization. Recently dysbiosis of the microbiota has been investigated as a potential contributor to the development of allergic sensitization and symptoms. We decided to review the current evidence and describe potential mechanisms between allergic rhinitis and microbial dysbiosis of the upper airway and GI tract.

\section{Methods:}

To perform our review, we utilized PubMed, compiling the most relevant and well-performed studies focusing on the gut and upper respiratory microbiota in allergic rhinitis. Association and experimental studies were predominantly sought and used, though systemic reviews and review articles were included to ensure we included all pertinent studies within the past ten years.

\section{Findings:}

Significant evidence exists supporting the hypothesis that dysbiosis in upper respiratory and gut microbiota is associated with the development of allergic rhinitis. Additionally, existing longitudinal studies demonstrate that dysbiosis appears to precede the development of allergic symptoms, while experimental studies in murine models have provided evidence for defining a causative role for microbial dysbiosis. To date, modulation of the microbiome shows efficacy for treatment, but little to no efficacy for prevention.

\section{Conclusion and Potential Impacts:}

This review highlights the potential role of the gut and upper respiratory microbiota in the development of allergic rhinitis, providing an additional avenue for treatment and prevention in clinical settings. Given the results of prevention studies utilizing microbiome modulation, more research is required to establish the exact role of the microbiota in allergic disease and to examine the effects of personalized formulations of probiotics to further our understanding and possibly prevent the development of allergic rhinitis. 